Signal \& Image Processing : An International Journal (SIPIJ) Vol.3, No.3, June 2012

\title{
A NOVEL APPROACH COLOURED OBJECT TRACKER WITH ADAPTIVE MODEL AND BANDWIDTH USING MEAN SHIFT ALGORITHM
}

\author{
S.A.Mohammadi ${ }^{1}$ and M.R. Mahzoun ${ }^{2}$ \\ ${ }^{1}$ Department of ICT Engineering, IHU University, Tehran, Iran \\ seyed_amir_mohammadi@yahoo.com \\ 2 Department of ICT Engineering, IHU University, Tehran, Iran \\ rezamahzoun@yahoo.com
}

\begin{abstract}
The traditional color-based mean-shift tracking algorithm is popular among tracking methods due to its simple and efficient procedure, however, the lack of dynamism in its target model makes it unsuitable for tracking objects which have changes in their sizes and shapes. In this paper, we propose a fast novel threephase colored object tracker algorithm based on mean shift idea while utilizing adaptive model. The proposed method can improve the mentioned weaknesses of the original mean-shift algorithm. The experimental results show that the new method is feasible, robust and has acceptable speed in comparison with other algorithms.
\end{abstract}

\section{KEYWORDS}

Adaptive bandwidth; mean shift algorithm; object tracking; target model updating; variable target model.

\section{INTRODUCTION}

Object tracking is one of the most important topics in computer vision and, yet, due to the presence of a lot of undesired phenomena such as noises, occlusions, clutters, changes in the foreground objects or in the background environment and so on, it is still a challenging subject of interest. To achieve an efficient visual object tracking method, a large number of algorithms such as mean-shift tracker, covariance tracker, particle filters, and so on are presented in real applications, while simple algorithms in this field have many demands [1-4].

Real-Time object tracking has many applications such as surveillance, perceptual user interfaces, augmented reality, smart rooms, object-based video compression and driver assistance [5-24].

Among different tracking algorithms, mean shift (MS) based methods have their special places because of their simplicity in computation while have good accuracies [25].

Mean shift is a nonparametric density estimator which iteratively computes the nearest mode of a sample distribution starting from a hypothesized mode [26].

Despite of its promising performance, simplicity and efficiency, many algorithms have been proposed to solve the various problems that mentioned before [27-36].

DOI : 10.5121/sipij.2012.3301 
Signal \& Image Processing : An International Journal (SIPIJ) Vol.3, No.3, June 2012

Developed methods have tried to solve three major problems of accurate target representation, dynamic tracking bandwidth, and target model updating, exist in trackers algorithms [37-41]. The classical mean shift tracking algorithm has three main restrictions, instead of first, the lack of accurate representation of target shows itself when the background and target region are highly correlated. In this case the localization accuracy of the object's tracking will be declined.

The second one, the constancy of the kernel bandwidth in classical mean shift, when the object scale changing during tracking and in practical experiences this constancy of bandwidth causes confliction of bandwidth size and object scale. Third limitation is constant target model while object appearance change during tracking. In practical experience appearance of target will be changed and if modeling of target be constant, certainly tracker will loss object. These limitations caused by inaccuracy of tracker's bandwidth or incorrect modeling of target area and constant target model during tracking.

Heretofore developed methods just try to solve one or two of these problem simultaneously [4249] and some of method introduced for tracking algorithms comparison [25], [50-52].

In this paper, we introduce Dynamic Mean Shift Tracker (DMST) which has adaptive bandwidth and target modeling, the object modeling and time of bandwidth will be updated during tracking dynamically.

In proposed method, accuracy in target modeling and adaptive bandwidth allows to have the target model adaptation with variable appearance of the target. Our proposed method solves the aforesaid problem of the original algorithms. Also we used novel approach for comparison of methods. The rest of this paper is as follows:

In Section 2, classical Mean-Shift Tracking introduced. In Section 3, we describe corrected background-weighted histogram (CBWH). The automated scale selection and target modeling adaptation are described sequentially in Section 4 and Section 5. We introduce DMST method In Section 6. In section 7, Description of new comparison parameters and experimental tracking results will be showed. Lastly, we give a conclusion in Section 8.

\section{Mean-Shift Tracking: An Overview}

In this section we introduce the Mean Shift tracker described in [26].

The Mean Shift tracker works by searching in each frame for the location of an image region whose colour histogram is closest to the reference colour histogram of the target [27].target features and way of modelling it, target candidates and geometric relations between target model and candidate are the main subject of the MS algorithm [25].

\subsection{Target features and modeling}

Although histograms are not the best nonparametric approximation of densities but they can be good criterions for target tracking [10]. Currently, a widely used target representation is the colour histogram because of its independence of scaling and rotation and its robustness to partial occlusions [26].

In the mean shift tracking algorithm, the colour (Probably Density Function) PDF of target location which is shown by $\mathrm{q}$ in colour space and weighted according to an isotropic kernel is target feature [25], pixels closer to the centre of the kernel are assigned greater weight than those near the boundary and the tracker compares two histograms using a metric based on the Bhattacharyya coefficient [52]. 
Signal \& Image Processing : An International Journal (SIPIJ) Vol.3, No.3, June 2012

Target candidate model in next frame, created by PDF of target candidate area with center of y and named $\mathrm{p}(\mathrm{y})$. Both PDFs (target and candidate), contains a good approximation of intrinsic feature of target [25].

For have fast computational processing some of histogram bins negligible. Created target and candidate model is defined as:

Target model:

$\left\{\hat{\mathrm{q}}_{\mathrm{u}}\right\} \mathrm{u}=1 \ldots \mathrm{m} \quad \sum_{\mathrm{u}=1}^{\mathrm{m}} \hat{\mathrm{q}}_{\mathrm{u}}=1$

Candidate model:

$$
\hat{\mathrm{p}}(\mathrm{y})=\left\{\hat{\mathrm{p}}_{\mathrm{u}}(\mathrm{y})\right\} \mathrm{u}=1 \ldots \mathrm{m} \sum_{\mathrm{u}=1}^{\mathrm{m}} \hat{\mathrm{p}}_{\mathrm{u}}=1
$$

Where $\hat{\mathrm{q}}_{\mathrm{u}}$ and $\hat{\mathrm{p}}_{\mathrm{u}}$ are normalized target and candidate models and $\mathrm{u}$ is colours exist in target and candidate locales. Using Equation (1), similarity of two models determines.

$$
\hat{\mathrm{p}}(\mathrm{y}) \equiv \rho[\hat{\mathrm{p}}(\mathrm{y}), \hat{\mathrm{q}}]
$$

In this equation $\hat{\mathrm{p}}(\mathrm{y})$ is measured similarity of two models and its local maximums indicate on similarity value between target candidate in new frame and the target in first frame[26].

\subsection{Target Modeling}

The most important step in coloured target tracking is to establish target colour distribution model, which provides a vital basis for weight updating and target state estimation [53]. The target model should be as discriminative as possible to distinguish between the complex target and background [54]. there are different approaches to building target models, Some use constant or adaptive colour threshold to distinguish the target from background, some model the intensity of each pixel of the target with a normal distribution, and others use a mixture of Gaussian distributions to model pixel values [53].

In practice, target model is displayed by a rectangular or circular domain. The function $\mathrm{b}: \mathrm{R}^{2} \rightarrow\{1 \ldots \mathrm{m}\}$ sets index $\mathrm{b}\left(\mathrm{x}_{\mathrm{i}}^{*}\right)$ corresponds to quantized histogram bar in colour space to a pixel locates at $x_{i}^{*}$. The values corresponding to existed colour is determines by [26]:

$$
\hat{q}_{u}=C \sum_{i=1}^{n} k\left(\left\|x_{i}^{*}\right\|^{2}\right) \delta\left[b\left(x_{i}^{*}\right)-u\right]
$$

Where $\hat{\mathrm{q}}_{\mathrm{u}}$ is the probability of uth element of $\hat{\mathrm{q}}_{\mathrm{u}}, \delta$ is kronecker delta function. The normalization coefficient, $\mathrm{C}$, under condition $\sum_{\mathrm{u}=1}^{\mathrm{m}} \hat{\mathrm{q}}_{\mathrm{u}}=1$ is defined as:

$$
\mathrm{C}=\frac{1}{\sum_{\mathrm{i}=1}^{\mathrm{n}} \mathrm{k}\left(\left\|\mathrm{x}_{\mathrm{i}}^{*}\right\|^{2}\right)}
$$

Using $\hat{q}_{u}$ three distributions of colour in RGB space, convert to a linear function that cause to simplicity of computational procedures.

\subsection{Target candidate modeling}

By assuming $\left\{\mathrm{X}_{\mathrm{i}}\right\} \mathrm{i}=1 \ldots \mathrm{n}_{\mathrm{h}}$ as the location of normalized target candidate pixels centered at $\mathrm{y}$ on current frame, Similar to target pixels, with using function $\mathrm{k}(\mathrm{x})$ that has comparable bandwidth size (h) like target's, these pixels weighted and the values for target candidate model related to colours $\mathrm{u}=1, \ldots \mathrm{m}$ can be computed as below[25]: 
Signal \& Image Processing : An International Journal (SIPIJ) Vol.3, No.3, June 2012

$$
\hat{\mathrm{p}}_{\mathrm{u}}(\mathrm{y})=\mathrm{C}_{\mathrm{h}} \sum_{\mathrm{i}=1}^{\mathrm{n} h \mathrm{~h}} \mathrm{k}\left(\left\|\frac{\mathrm{y}-\mathrm{x}_{\mathrm{i}}}{\mathrm{h}}\right\|^{2}\right) \delta\left[\mathrm{b}\left(\mathrm{X}_{\mathrm{i}}\right)-\mathrm{u}\right]
$$

Where $C_{h}$ is normalization coefficient ( $y$ independent), computed by [54]:

$$
C_{h}=\frac{1}{\sum_{i=1}^{n h} k\left(\left\|\frac{y-x_{i}}{h}\right\|^{2}\right)}
$$

\subsection{Bhattacharyya coefficients}

In classical mean shift tracking, similarity between target and a candidate computed by the Bhattacharyya coefficient

Using Equation that defined as below [26]:

$$
d(y)=\sqrt{1-\rho[\hat{p}(y), \hat{q}]}
$$

In this Equation $\rho[\hat{\mathrm{p}}(\mathrm{y}), \hat{\mathrm{q}}]$ computed by Equation (7).

$$
\hat{\mathrm{p}}(\mathrm{y}) \equiv \rho[\hat{\mathrm{p}}(\mathrm{y}), \hat{\mathrm{q}}]=\sum_{\mathrm{u}=1}^{\mathrm{m}} \sqrt{\hat{\mathrm{p}}_{\mathrm{u}} \hat{\mathrm{q}}_{\mathrm{u}}}
$$

In computational theory if target and candidate model have a perfect matching, the value of (7) will be one. Comprehensive description about Bhattacharyya coefficients can be found in [10].

\subsection{Target localization in image sequence}

Whatever distance (6) is going to minimization, similarity metric (7) rising to maximization. The tracking process starts from a point (and its vicinity) which it was the target place in previous frame and continue until the mentioned distance reach to predefined minimum (Equation 6) or maximum iteration happens[25].

\section{CORRECTED BWH (CBWH)}

In classical mean shift algorithm, PDF of image colour use to characterization of the statistical distribution of image in colour space. The conventional PDF of image is sensitive to noisy interference such as lighting changes and quantization errors and similarity with background.

Comaniciu et al [26], proposed a simple representation of the background features, and used it for selecting only the salient parts from the representations of the target model and target candidates that called Background-Weighted Histogram (BWH).

Thereafter, Ning et al. [55] proved that the BWH practically does not developed mean shift tracking and Its result is precisely the same as that without using $\mathrm{BWH}$, in the other word $\mathrm{BWH}$ does not introduce any new information because the mean shift iteration formula is invariant to the scale transformation of weights.

In [55], Ning et al. proposed a corrected BWH (CBWH) and shown that the performance of BWH algorithm will increase if Equation (8) used for target modeling in the first frame and Equation (4) used for target candidate modeling in image sequences [55].

$$
\left\{\mathrm{v}_{\mathrm{u}}=\min \left(\frac{\hat{\mathrm{o}}^{*}}{\hat{\mathrm{o}}_{\mathrm{u}}}, 1\right)\right\}_{\mathrm{u}=1 \ldots \mathrm{m}}
$$


Where ${ }^{\mathrm{v}} \mathrm{u}$ is transfer function and $\hat{o}_{\mathrm{u}}$ is background histogram, it is this algorithm known as CBWH.

Equation (8) is a transformation that used for decreasing the effect of background colour on target model calculated by Equation (9).

$$
\hat{\mathrm{q}}_{\mathrm{u}}^{\prime}=\mathrm{C}^{\prime} \mathrm{v}_{\mathrm{u}} \sum_{\mathrm{i}=1}^{\mathrm{n}} \mathrm{k}\left(\left\|\mathrm{x}_{\mathrm{i}}^{*}\right\|^{2}\right) \delta\left[\mathrm{b}\left(\mathrm{x}_{\mathrm{i}}^{*}\right)-\mathrm{u}\right]
$$

Where

$$
C^{\prime}=\frac{1}{\sum_{i=1}^{n} k\left(\left\|x_{i}^{*}\right\|^{2}\right) \sum_{u=1}^{m} v_{u} \delta\left[b\left(x_{i}^{*}\right)-u\right]}
$$

In CBWH algorithm, first of all, the target background ${ }^{o} \mathrm{u}$ is calculated then by using Equation (8) transfer function ${ }^{\mathrm{v}} \mathrm{u}$ prepared.

In proposed method, we used CBWH algorithm to reduction of interference impact, between background and target information for improve robustness of target localization.

\section{Automated SCAlE SEleCtion}

The performance of the mean shift algorithm strongly depends on the kernel bandwidth parameters [56], which determine region of interest for tracking and many developed algorithms to solve this problem.

In the classical mean shift algorithm and introduced method in [55] assumed that the object scale during the course of tracking is constant but in real world, and in practice, it is not uncommon to observe objects with complex shapes whose scale and orientation constantly change due to the camera and object motions [57]. Tracking algorithm shall be able to adjust the tracking bandwidth in real time when the size of the tracked objects changes frequently.

We use size adaptation method proposed in [58], in this method with using a new multi-scale information measurement that named MSIIM, automatically bandwidth updated. Qian et al. [58] proved that the distance between the observer and the object was reflected by the MSIIM and this feature has good performance to adjustment of bandwidth. The updating process is described as follows [58]:

In proposed method in [58], periodically, MSIIMs of target area compared with previous computed MSIIMs, and according to the change of the MSIIMs, size of bandwidth updated.

At the first frame of images sequence, in current bandwidth size MSIIM calculated and named I1 and in multiply factor of $1+\alpha$ on the tracking bandwidth, the MSIIM calculated and named $\mathrm{I}_{2}$ (Figure1.(a)), at Nth frame MSIIMs calculated as same way and named, $\mathrm{I}_{4}$ and $\mathrm{I}_{5}$. The same process is also performed on the $\mathrm{n}+\mathrm{N}$-th frames to scale updating, In Nth if $\mathrm{I}_{5}-\mathrm{I}_{2} \geq 0$, this means, target scale is decreasing (Figure1.(b)), and with using Equation (11) update scale changing factor $\mathrm{S}$ calculated.

In other hand, if $I_{5}-I_{2}<0$, this means, target scale is increasing (Figure1.(c)), and with using Equation (12) update scale changing factor $\mathrm{S}$ calculated. 
Signal \& Image Processing : An International Journal (SIPIJ) Vol.3, No.3, June 2012

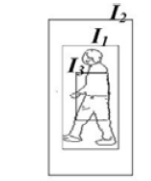

(a) current frame

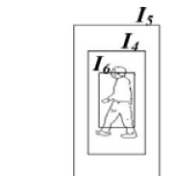

(b)video sequence with zoom-out object

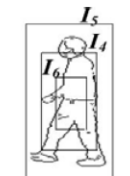

(c) video sequence with zoom-in object

Figure 1. Relationship between MSIIM and window scale

$$
\begin{aligned}
& S=\left\{\begin{array}{lc}
\lg \left(\beta-\frac{I_{5}-\left(I_{2}-I_{1}\right)}{I_{1}}\right) & \frac{I_{5}}{I_{4}}>\frac{I_{2}}{I_{1}} \\
0 & \text { Others }
\end{array}\right. \\
& S=\left\{\begin{array}{lc}
\beta .\left(\lg \frac{I_{4}}{I_{1}}\right) & I_{1} .0 .95>I_{4} \\
0 & \text { Others }
\end{array}\right.
\end{aligned}
$$

Parameter $\beta$ In Equations (11) and (12), used for elimination of background effect, and is dependent on video component. With using obtained the scale factor $\mathrm{S}$, the height $(\mathrm{H})$ and width (W) of the tracking window according to Equation (13) updated.

$$
S=\left\{\begin{array}{l}
H=H \cdot(1+S) \\
W=W \cdot(1+S)
\end{array}\right.
$$

Although, the proposed method in [58] has good results for scale updating but due to using the classical colour histogram based mean shift tracking framework when clutters and occlusions in the appearance model accrued, it has a poor tracking results.

We integrate CBWH method proposed in [55] with [58] to solve this problem.

Qian et al. [58], use a constant period of bandwidth updating, in practice observed when bandwidth size becomes very big tracker performance decreased and if bandwidth size becomes very small, tracker loss target, to solve this problem, in our proposed method, using number of size updating in side of increasing or decreasing of bandwidth, this period became variable.

In proposed method, we define two new parameters to limitation of unnecessary scale size updating, in proposed approach, if we have a scale size increasing, one unit will be deduced from defined limitation parameter for scale size decreasing and one unit will be added to limitation parameter of scale size increasing and if we have a scale size decreasing, one unit will be deduced from defined limitation parameter for scale size increasing and one unit will be added to limitation parameter of scale size decreasing. While number of scale changing is equal to defined limitation, for prevent of unnecessary scale updating, updating procedure stopped. Using this method, bandwidth updating period become variable and the scale changing velocity become depend on the scene of the video.

In our developed method, instead of proposed method in [58], that the value of parameter $\alpha$ is changed over tracking, in our method, this value is assumed constant.

In scenarios that the size of target changes slowly and the background has same colour, if $\alpha$ has been variable, due to use procedure introduced in [58] and because of similarity between target 
and background, we have a miscalculation in the tracked object's MSIIM feature that affected by incorrect window scale.

In proposed method, the value of parameter $\alpha$ is constant and the practical results showed reasonably of tracking bandwidth during tracking.

\section{TEMPLATE UPDATE}

The goal of template update is to allow the appearance of the reference image to change in order to reduce feature drift while at the same time preventing spatial drift from entering the system [59]. S.lankton at el. [59] proposed a periodically method, the key model $\tilde{\mathrm{M}}$ is updated every $\mathrm{N}$ frames with the procedure shown in Figure 2.

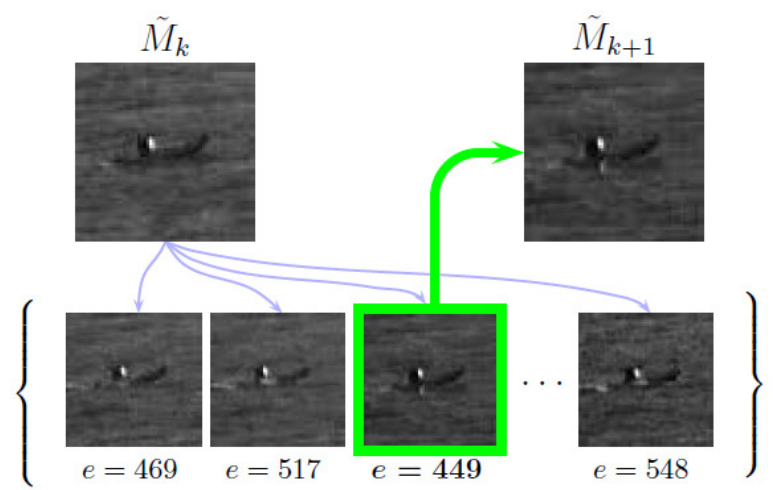

Figure 2. S.lankton at el. update the key model $\tilde{\mathrm{M}}_{\mathrm{k}}$ periodically to prevent feature drift. The new key model $\tilde{\mathrm{M}}_{\mathrm{k}+1}$ is selected by choosing a model from the $\operatorname{set}\left\{\hat{\mathrm{M}}_{\mathrm{i}}\right\}_{i=1}^{\mathrm{N}}$ that has the lowest error e when compared to the previous key model $\tilde{\mathrm{M}}_{\mathrm{K}}[59]$.

During the template matching step, the past $\mathrm{N}$ models when size of bandwidth changing, collected and denoted as $\left\{\hat{\mathrm{M}}_{\mathrm{i}}\right\}_{\mathrm{i}=1}^{\mathrm{N}}$. The new key model, $\tilde{\mathrm{M}}_{\mathrm{K}+1}$ is chosen as the best representative of this set as determined by a matching error. They used Equation (14) for matching error is similarity between key model and registered models:

$$
\mathrm{e}(\hat{\mathrm{M}}, \tilde{\mathrm{M}})=\|\hat{\mathrm{M}}-\tilde{\mathrm{M}}\|^{2}
$$

In proposed method, above procedure used with small differences, to similarity comparison, Equation (14) replaced by Bhattacharayya coefficient and with using number of iteration of size changing in one way (increasing or decreasing),constant checking time period of template updating in [59] being dynamic, also threshold of template updating will be replaced by similarity comparison of new template and initialized template (related Bhattacharayya coefficient), in this way not only checking of template updating time being dynamic but also threshold of template updating will be automatically updated. In proposed method, according to the separation of the foreground and background distributions by using method introduced in [55], target template updated and in new method instead of [59], bandwidth size is variable.

\section{DMST PROCEDURE}

We integrate the methods described in Ref. [58] and [59] into the CBWH tracking framework introduced in [55] and call this new tracker as Dynamic Mean Shift Tracker (DMST) which has 
Signal \& Image Processing : An International Journal (SIPIJ) Vol.3, No.3, June 2012

adaptive bandwidth and robustness target representation and its target template will be updated dynamically.

The DMST algorithm is described as follows:

1. Create target model according to CBWH method [55] and calculate MSIIMs, $\mathrm{I}_{1}(\mathrm{n})$ and $\mathrm{I}_{2}(\mathrm{n})$;

2. Set frame number $n=0$, scale factor $S=0$, template updating time factor $D=0$ and the processing interval to $\mathrm{N}$;

3. Fetch a video frame, and set $n=n+1$. If $n=1$, initialize the tracker and the height $\mathrm{H}$ and width $\mathrm{W}$ of the tracking window; otherwise, go to next step;

4. If frame number $\mathrm{n}$ is a multiple of $\mathrm{N}$, then go to step (5), or else, set $\mathrm{S}=0$, and go directly to (6);

5. Calculate MSIIM, $\mathrm{I}_{4}(\mathrm{n})$ and $\mathrm{I}_{5}(\mathrm{n})$ respectively on the current frame. According to the information change, determine the object change tendency. Then use Equations (11) and (12) to obtain the scale factor $S$, and set $I_{2}(n)=I_{5}(n), I_{1}(n)=I_{4}(n)$. If $S$ isn't equal to zero, store related model and set $\mathrm{D}=\mathrm{D}+1$. If $\mathrm{D}$ is equal to proposed limit then compare similarity between initial target model and stored models and if similarity is equal or less than threshold, replace target model that created according Equitation (9) by related stored model and change threshold by calculated differences between similarity of new and old target model;

6. Use Equation (13) to update the height and width. If the video does not finish yet then go back to step 3. Otherwise exit the algorithm.

Results show that DMST can lead to faster convergence and more accurate localization than methods introduced in [55, 58-59] and Even if the target is not well initialized, the proposed algorithm can still correctly track the target, which is difficult to perform by the mentioned target representation methods.

\section{COMARISION METHOD}

Evaluation of tracking algorithms is a challenging topic especially when the results of these methods are close to each other [25, 60-62]. Here we used two parameters which are extracted from the structure of classical mean shift algorithm and introduced in [25]. Instead of first is the mean of confidence coefficient (MCC) shown in Equation (14):

$$
\mathrm{MCC}=\frac{\sum_{\mathrm{i}=1}^{\mathrm{n}} \mathrm{CC}_{\mathrm{i}}}{\mathrm{n}}
$$

Where

$$
\mathrm{CC}_{\mathrm{n}}=\frac{\sum_{\mathrm{i}=1}^{\mathrm{x}}\left(\mathrm{MS}(\mathrm{i})_{\mathrm{n}}-\text { MinDist }\right)}{\mathrm{x}}
$$

In this Equation $\mathrm{CCn}$ is the confidence coefficient in frame $\mathrm{n}$ and indicates the mean distance between the center of target candidate in frame $\mathrm{n}$ and process exit criterion (MinDist), MS in frame $\mathrm{n}$ is the difference between the centers of target candidate (found i time in current frame) and target (found in frame $\mathrm{n}$ of previous try) [25].

The second parameter, $\mathrm{NV}$, is the normalized variance of $\mathrm{CCn}$ with respect to $\mathrm{MCC}$, defined as: 
Signal \& Image Processing : An International Journal (SIPIJ) Vol.3, No.3, June 2012

$$
\mathrm{NV}=\frac{\sum_{\mathrm{i}=1}^{\mathrm{n}}\left(\mathrm{CC}_{\mathrm{i}}-\mathrm{MCC}\right)^{2}}{\mathrm{n}}
$$

If the MS distance was smaller than MinDist the center of target candidate (correspond to MS) will be selected as new target position, else, this position is used in place of target center in previous frame to compute the target position in current frame. The mean of target candidates distances from MinDist computed by Equation (16), represents the accuracy of tracking process on that frame. Variance is used to show the reliability of the method. The smaller values indicate on more repeatability of the results [25].

For comparison of our method with original algorithms we use both introduced parameters in [25] and conventional parameters like number of iterations and visual output.

\section{EXPERIMENTAL RESULTS}

In this section, DMST algorithm tested by using two video sequences and results compared with origin developed mean-shift algorithms and classical mean shift algorithm. All algorithms were implemented under the programming environment of MATLAB 9.2a. In all of the experiments, the RGB colour model was used as the feature space and it was quantized into $16 \times 16 \times 16$ bins.

In our experiments two segments of videos were subjected to test, and the tracking window was set to rectangle. Figure 3 shows the target areas which are tracked in videos.

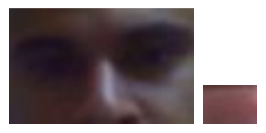

Figure 3. Target areas which are tracked in videos

The first experiment is on the pink sequence (the tracking results in Figure 4 and the statistics in Table I), which was used in [55], [58], [59]. This sequence has 1013 frames of spatial resolution $352 \times 240$. The target is the head of pen that moves quickly and rescaled during tracking. As it is shown in Fig. 4(a), we initialized the target model (inner white rectangle). In this sequence, the colour of (pink) the target is very similar to the background and thus some target features in the middle of sequence are presented in the background. Experimental results in Figure 4 show that the Mean shift tracker, CBWH lose the object, while the proposed DMST and proposed method in [58] successfully tracks the object over the whole sequence, most important different between DMST result and proposed method in [58] is updating of target model in DMST (figure 5), this adaptation cause to accuracy of tracking (Table I).

The second experiment is on the Face sequence (the tracking results in Figure 6 and the statistics in Table I), which was used in [55], [58], [59]. This sequence has 661 frames of spatial resolution $352 \times 240$. The target is the Face that it moves quickly and screen is noisy. In this sequence, the Face is rescaled during the frames and covert by similar colour hand. Experimental results in Figure 6 show that the Mean shift tracker and CBWH algorithms can't adaptive themselves with scale changing, while the proposed method in [58] and DMST are successfully adapted with changes. although as shown in Figure 6.(c) proposed method in [58] can't locate target area robustness due to insufficient target modeling; but as shown in Figure 6.(d), due to target model updating that cause to good target localization and tracking (refer to Figure 7), proposed Method has a excellent output,

As shown in Graphs 1 and 2, threshold of target model updating, after target model replacement changed, new threshold will be use in the next model adaptation. 
Signal \& Image Processing : An International Journal (SIPIJ) Vol.3, No.3, June 2012

The computational cost will be reducing using $\mathrm{C}$ language and the speed of tracking will be faster than using MATLAB.

Table I illustrate the stochastic results of the above tests. The values of MCC, NV, and iteration. For each of the four algorithms for both pink and Face sequences are collected in this table. As it is shown in Table I, the proposed method has good speed and the visual presentation in figures show robustness of DMST in comparison with other algorithms.

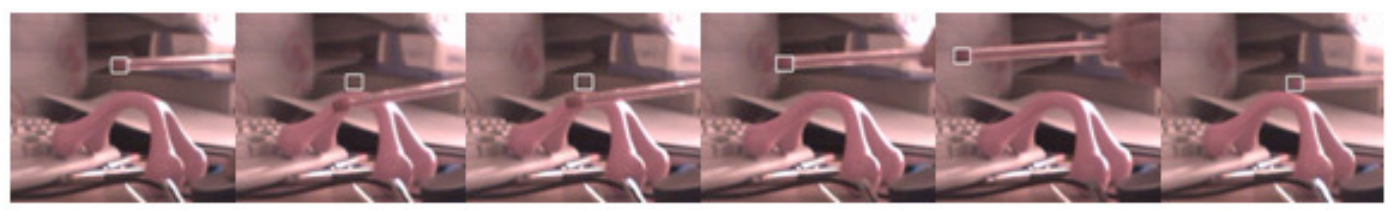

(a): Mean shift tracking results on the pink sequence. Frames left to right $1,335,380,628,729,985$ are displayed.

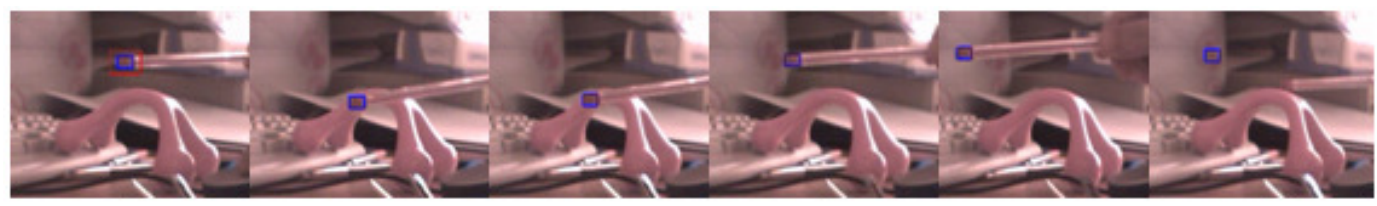

(b): CBWH tracking results on the pink sequence. Frames left to right $1,335,380,628,729,985$ are displayed.

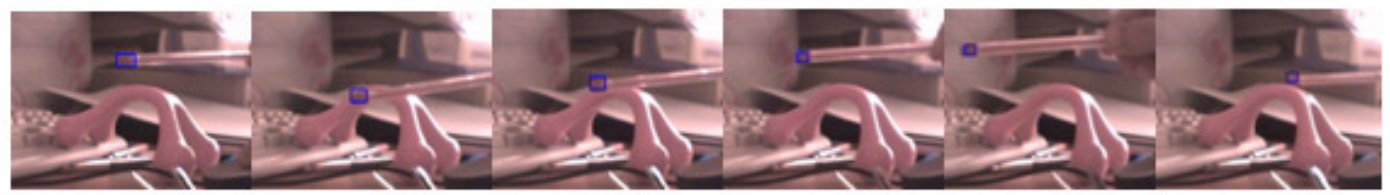

(c): Method [58] tracking results on the pink sequence. Frames left to right 1, $335,380,628,729,985$ are displayed.

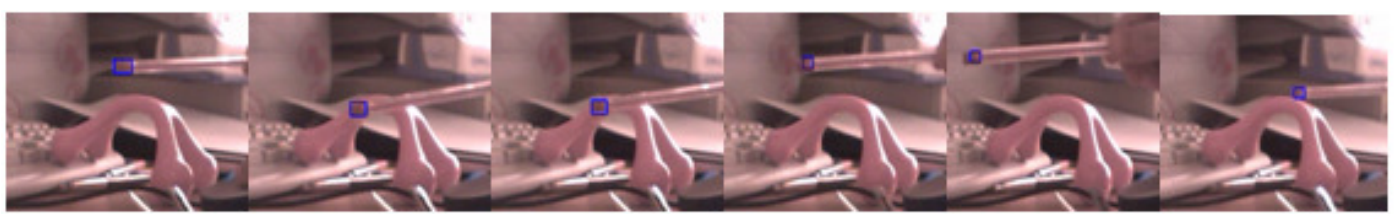

(d): DMST tracking results on the pink sequence. Frames left to right 1, 335,380,628,729,985 are displayed.

Figure 4. Results on pink sequence 
Signal \& Image Processing : An International Journal (SIPIJ) Vol.3, No.3, June 2012

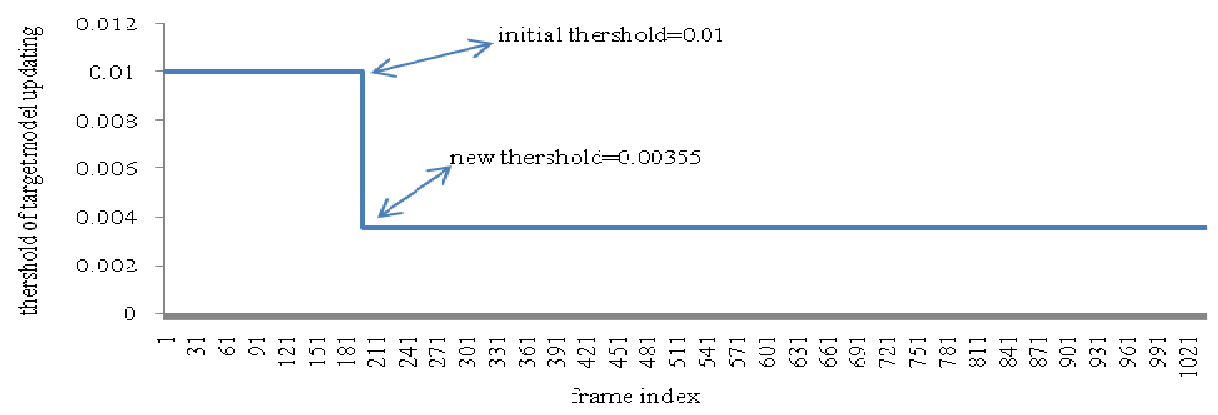

Graph 1. during terget template changing, Thershold of target model updating will be updated.

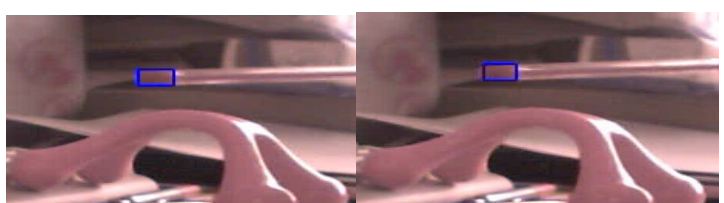

Figure 5. intial target template(right) replaced by new target template (right).

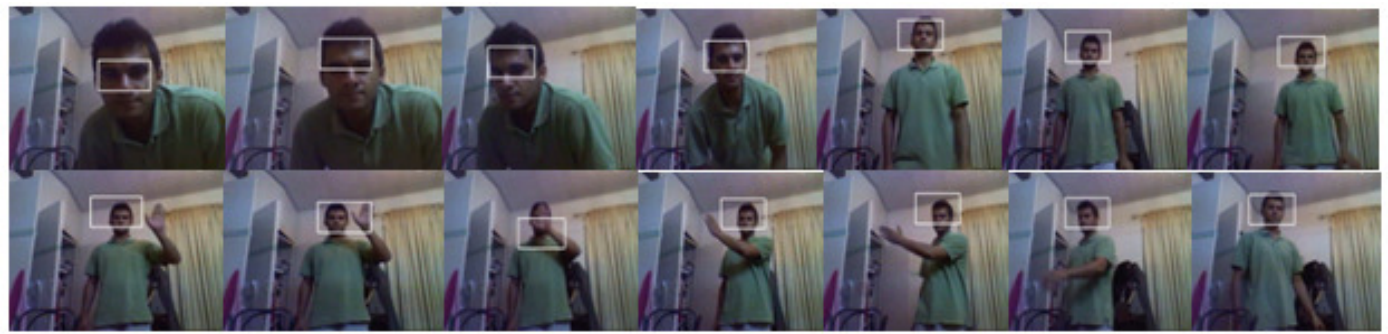

(a): Mean shift tracking results on the Face sequence. Frames left to right(upper) 1,19,50,164,203,251,304 and (bottom) 357,368,413,500,531,569,668 are displayed.

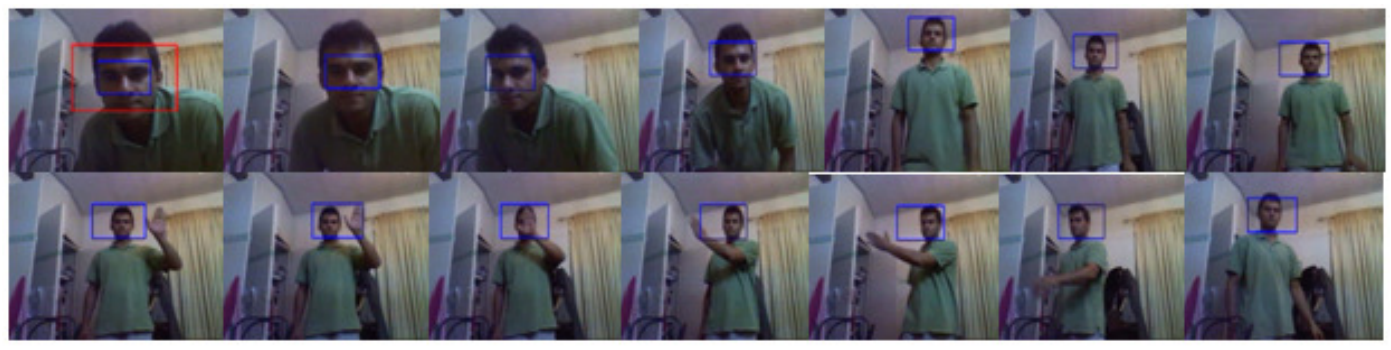

(b): CBWH tracking results on the Face sequence. Frames left to right(upper) $1,19,50,164,203,251,304$ and (bottom) 357,368,413,500,531,569,668 are displayed.

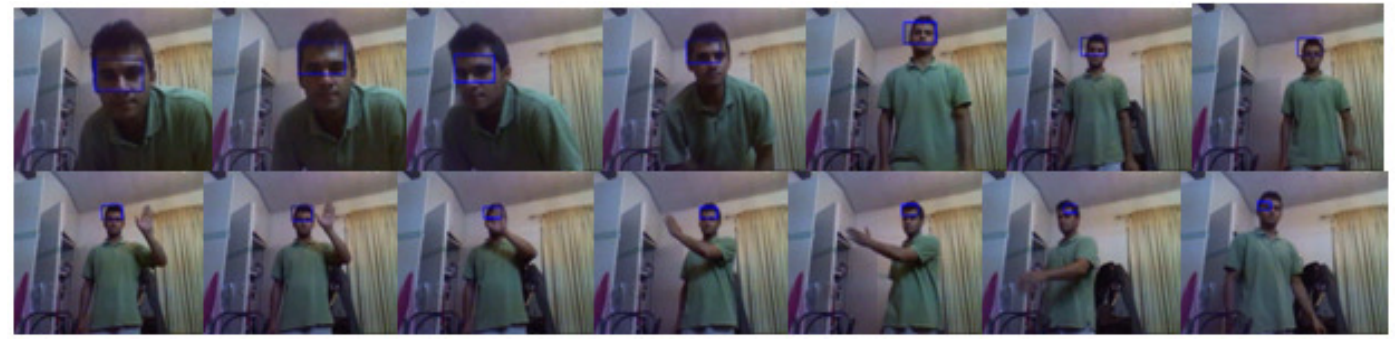

(c): Method [58] tracking results on the table tennis player sequence. Frames left to right(upper) $1,19,50,164,203,251,304$ and (bottom) 357,368,413,500,531,569,668 are displayed. 
Signal \& Image Processing : An International Journal (SIPIJ) Vol.3, No.3, June 2012

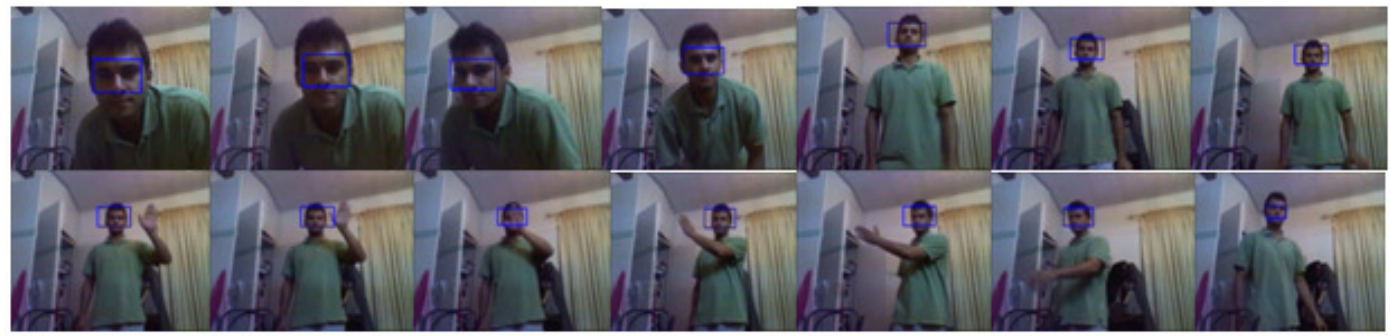

(d): DMST tracking results on the Face sequence. Frames left to right(upper) $1,19,50,164,203,251,304$ and (bottom) 357,368,413,500,531,569,668 are displayed.

Figure 6. Results on the Face sequence

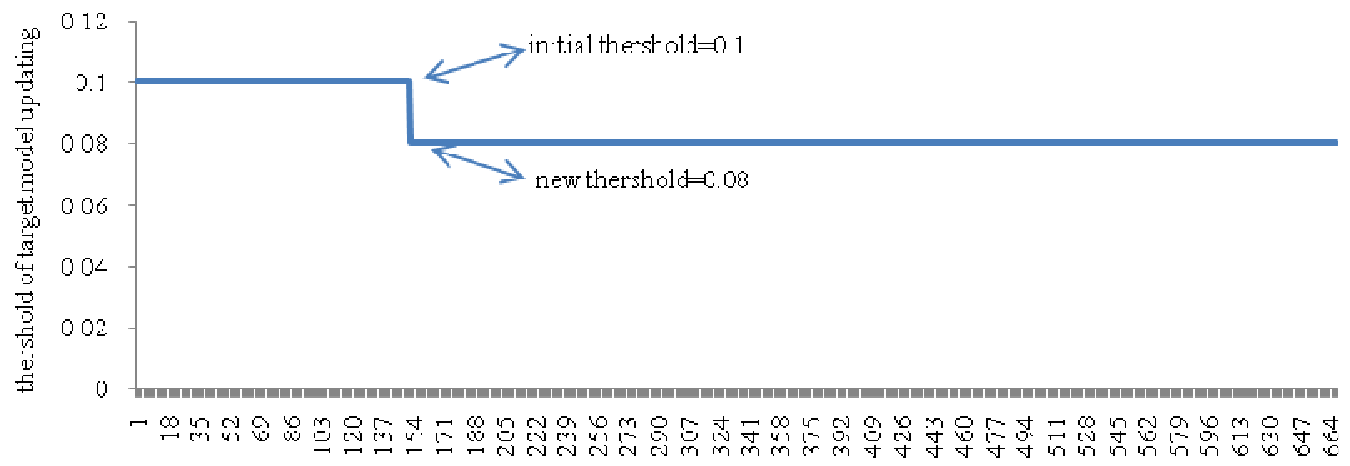

frame index

Graph 2. during terget template changing, Thershold of target model updating will be updated.

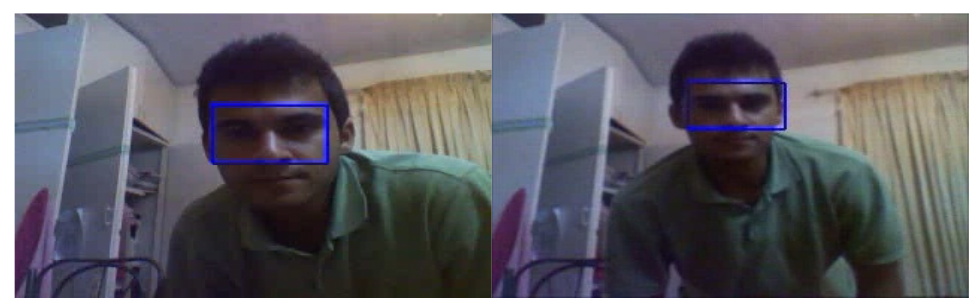

Figure 7. intial target template(right) replaced by new target template (right).

Table 1. comarition of the mean-shift based algorithms and prpoposed method on the two sequences

\begin{tabular}{|c|c|c|c|c|}
\hline SEQUeNCES & ALGORITHMS & MCC & NV & ITERATION \\
\hline \multirow{5}{*}{ PINK } & Mean-shift & 0.0262 & $4.0371 \mathrm{e}-04$ & 3.0142 \\
\cline { 2 - 5 } & CBWH & 0.024367 & $5.1565 \mathrm{e}-04$ & 2.6413 \\
\cline { 2 - 5 } & Self-adapt & 0.022911 & $3.2296 \mathrm{e}-04$ & 3.0116 \\
\cline { 2 - 5 } & DMST & 0.026237 & $4.3519 \mathrm{e}-04$ & 3.1849 \\
\hline \multirow{4}{*}{ FACE } & Mean-shift & 0.033108 & $3.29 \mathrm{e}-04$ & 3.507 \\
\cline { 2 - 5 } & CBWH & 0.031374 & $4.239 \mathrm{e}-04$ & 3.4701 \\
\cline { 2 - 5 } & Self-adapt & 0.034304 & $3.462 \mathrm{e}-04$ & 3.3144 \\
\cline { 2 - 5 } & DMST & 0.032629 & $3.292 \mathrm{e}-04$ & 3.1781 \\
\hline
\end{tabular}


Signal \& Image Processing : An International Journal (SIPIJ) Vol.3, No.3, June 2012

\section{CONCLUSION AND Future Directions}

In this paper, a feasible tracking algorithm with adaptability to scale and appearance changes of target and improve the target localization for real-time processing introduced. Dynamic Mean Shift Tracker (DMST) has adaptive bandwidth and target model. In addition our method solves the problems of original algorithms. The proposed method can be used in many tracking scenarios and it can be integrated with many existing tracking frameworks. Accuracy of bandwidth and introduced parameters such as In and number of size changing of bandwidth used for adaptation of target model during tracking. Bandwidth size changing and In changing behavior utilized for automation of control parameters used in algorithm of method [58].

\section{REFERENCES}

[1] Jwu-Sheng Hu,"3D object Tracking Using Mean-Shift and Similarity-Based Aspect-Graph Modeling",The 33rd Annual Conference of the IEEE Industrial Electronics Society (IECON) Nov. 58, 2007, Taipei, Taiwan.pp.2383-2388]

[2] Y.Wang, "Fuzzy Colour Histogram Based Kernel Tracking under Varying Illumination", Fourth International Conference on Fuzzy Systems and Knowledge Discovery (FSKD 2007)0-7695-28740/07 \$25.00 @ 2007 IEEE.]

[3] P.Pan, F.Porikli, "A new method for tracking performance evaluation based on a reflective model and perturbation analysis.", ICASSP, pp.3529-3532, 2009.]

[4] G.Lihua,Z,Yuanjian ,"Video Object Tracking Method Based on Snake Model Using Object's Histogram",0-7803-9584-0/06/\$20.00(2006)IEEE.

[5] V.Kettnaker,R.Zabih,"Bayesian multi-camera surveillance, "in Proc. IEEE Conf. on Computer Vision and Pattern Recognition, Fort Collins, CO, 1999, pp. 253-259.

[6] R.Collins, "Algorithms for cooperative multisensor surveillance", Proceedings of the IEEE, vol. 89, no. 10, pp. 1456-1477, 2001.

[7] M.Greiffenhagen, D.Comaniciu, "Design, analysis and engineering of video monitoring systems: An approach and a case study", Proceedings of the IEEE, vol. 89, no. 10, pp. 1498-1517, 2001.

[8] L.Li, "An Efficient Sequential Approach to Tracking Multiple Objects through Crowds for Real-Time Intelligent CCTV Systems", IEEE transactions on system, man, and cybernetics—part B: cybernetics, vol. 38, no. 5, October 2008.

[9] P.Bai, "Person-Tracking with Occlusion Using Appearance Filters", Proceedings of the 2006 IEEE/RSJ International Conference on Intelligent Robots and Systems October 9 - 15, 2006, Beijing, China.

[10] J. Krumm, S. Harris, B. Meyers, B. Brumitt, M. Hale, "Multi-camera multi-person tracking for Easy Living", in Proc. IEEE Intl. Workshop on Visual Surveillance, Dublin, Ireland, 2000, pp. 3-10.

[11] C. Wren, A. Azarbayejani, T. Darrell, and A. Pentland, "Pfinder: Real-time tracking of the human body", IEEE Trans. Pattern Anal. Machine Intell, vol. 19, pp. 780-785, 1997.

[12] J. Garcia, J. Valdivia, and X. Vidal, "Information theoretic measure for visual target distinctness", IEEE Trans. Pattern Anal. Machine Intell, vol. 23, no. 4, pp. 362-383, 2001.

[13] X. Li, M. Porikli," hidden markov model framework for traffic event detection using video features", IEEE, International Conference on Image Processing, ICIP, 2004.

[14] H. Veeraraghavan, "Combining Multiple Tracking Modalities for Vehicle Tracking at Traffic Intersections", New Wan, April 2004.

[15] Y. Wu, F. Lian, T. Chang, "Traffic Monitoring and Vehicle Tracking using Roadside Cameras", IEEE International Conference on Systems, Man, and Cybernetics October 8-11, 2006, Taipei, Taiwan.

[16] C.Picus, C.Beleznai, "Kernel-based storm tracking in radar data".

[17] G. R. Bradski, "Computer vision face tracking as a component of a perceptual user interface"in Proc.IEEE Workshop on Applications of Computer Vision, Princeton, NJ, October 1998, pp. 214 219.

[18] P.Vadakkepat,"Multimodal Approach to Human-Face Detection and Tracking", IEEE transaction on industrial electronics, vol. 55,no. 3, March 2008.

[19] A. D. Bue, D. Comaniciu, V. Ramesh, and C. Regazzoni, "Smart cameras with real-time video object generation", in Proc. IEEE Intl. Conf. on Image Processing, Rochester, NY, volume III, 2002, pp.429-432. 
Signal \& Image Processing : An International Journal (SIPIJ) Vol.3, No.3, June 2012

[20] C. LIEBE, "Accuracy Performance of Star Trackers-A Tutorial", IEEE transactions on aerospace and electronic system,vol. 38,no. 2 APRIL 2002.

[21] C. Zhang, C. Chen and X. Shen, "Boltzmann entropy-based guide star selection algorithm for star tracker", electronics letters 22nd January 2004 Vol. 40 No. 2.

[22] K.Zhang, "Research on the Image Matching and Tracking Algorithm for the End of Infrared Target Tracking", ICALIP, 2008.

[23] G.Huang, J.Su, "A Real-time Face Detection and Tracking", ICALIP, 2008.

[24] C. Lerdsudwichai , "Algorithm for Multiple Faces Tracking", ICME 2003.

[25] S.A. Mohammadi, R.mahzoun, "Introducing new parameters to compare the accuracy and reliability of mean-shift based tracking algorithms", Signal \& Image Processing : An International Journal (SIPIJ) Vol.2, No.3, ,pp.47-53, September 2011.

[26] D. Comaniciu, V. Ramesh and P. Meer, "Kernel-Based Object Tracking", IEEE Trans. Pattern Anal. Machine Intell., vol. 25, no. 2, pp. 564-577, 2003.

[27] A. Soto, "Self adaptive particle filter", in Proc. Int. Joint Conf. Artif. Intell, 2005, pp. 1398-1403.

[28] Y. Rui and Y. Chen, "Better proposal distributions: Object tracking using unscented particle filter", in Proc. IEEE Conf. Computer. Vis. Pattern Recognition., 2001, pp. 786-793.

[29] N. Bouaynaya and D. Schonfeld, "Complete system for head tracking using motion-based particle filter and randomly perturbed active contour", Proc .SPIE, vol. 5685, pp. 864-873, 2005.

[30] R. Merwe, A. Doucet, N. Freitas, and E. Wan, "The unscented particle filter", Technical Report cued/f-infeng/tr 380, Cambridge University Engineering Department, 2000.

[31] J.Shao, "Shape-regulated particle filtering for tracking non-rigid objects", ICIP 2006.

[32] G. Hager and P. Belhumeur, "Real-time tracking of image regions with changes in geometry and illumination", in Proc. IEEE Conf. on Computer Vision and Pattern Recognition, San Francisco, CA,1996, pp. 403-410.

[33] L.Zhang, "Real Time Mean Shift Tracking Using the Gabor Wavelet", Proceedings of the 2007 IEEE International Conference on Mechatronics and Automation August 5 - 8, 2007, Harbin, China.

[34] J. J. Pin, "tracking of objects in image sequences using bandwidth matrix mean shift algorithm", ICSP'O4 Proceedings.

[35] J.JIA, "tracking of objects in image sequences using multi-freeness mean shift algorithm", Proceedings of the Fourth International Conference on Machine Learning and Cybernetics, Guangzhou, 18-21 August 2005.

[36] S.Yonemoto, M.Sato, "Multi target Tracking Using Mean-shift with Particle Filter based Initialization",1550-6037/08, IEEE DOI 10.1109/IV, 2008.43.

[37] D. Liang, "Mean-Shift blob tracking with adaptive feature selection and scale adaptation", ICIP, 2007.

[38] Z.JIANG, "An improved mean shift tracking method based on nonparametric clustering and adaptive bandwidth", Proceedings of the Seventh International Conference on Machine Learning and Cybernetics, Kunming, pp.12-15, July 2008.

[39] I. Fidaner ,"Combined Colour and Texture Tracking for Video Post-Editing",978-1-4244-28816/08/\$25.00,2008, IEEE.

[40] D. Comaniciu, V. Ramesh, P. Meer, "The variable bandwidth mean shift and data driven scale selection"و Proc. of the IEEE Int'l Conf. on Computer Vision, 2001, pp. 438-445.

[41] Y.Shi,H.Liu, "adaptive feature-spatial representation for mean shift tracker",ICIP 2008.

[42] Peng NS, Yang J, Liu Z.: Mean Shift blob tracking with kernel histogram filtering and hypothesis testing. Pattern Recognition Letters. 26 (2005),pp. 605-614.

[43] G.Sen, "An adaptive weight values updating mean shift tracking algorithm", Proceedings of the 2009 IEEE International Conference on Information and Automation June 22 -25, 2009, Zhuhai/Macau, China.

[44] P.Li, "An Adaptive Binning Colour Model for Mean Shift Tracking", IEEE transactions on circuits and systems for video technology, vol. 18, no. 9, September 2008.

[45] Z.JIANG, "an adaptive mean shift tracking method using multiscale images", Proceedings of the 2007 International Conference on Wavelet Analysis and Pattern Recognition, Beijing, China, 2-4 Nov. 2007 .

[46] M.Jeong, "Adaptive Mean-Shift Tracking with Novel Colour Model", Proceedings of the IEEE International Conference on Mechatronics \& Automation Niagara Falls, Canada • July 2005.

[47] R.Collins, "Online Selection of Discriminative Tracking Features", IEEE transactions on pattern analysis and machine intelligence, VOL. 27, NO. 10, OCTOBER 2005.

[48] Peng NS, Yang J, Zhou DK, Liu Z, "Mean-Shift tracking with adaptive model update mechanism", Journal of Data Acquisition \& Processing. 20 (2005), pp. 125-129, Chinese. 
Signal \& Image Processing : An International Journal (SIPIJ) Vol.3, No.3, June 2012

[49] B.Hun, L.Duvis, "object tracking by adaptive feature extraction", 2004, International Conference on Image Processing (UP).

[50] P.Pan,F.Porikli,"A new method for tracking performance evaluation based on a reflective model and perturbation analysis."ICASSP 2009.

[51] F.Tung, "Efficient Target Recovery Using STAGE for Mean-shift Tracking",2009 Canadian Conference on Computer and Robot Vision,pp.16-22,2009.

[52] D.Caulfield,K.Dawson,"Evaluation of Multi-Part Models for Mean-Shift Tracking”, International Machine Vision and Image Processing Conference.pp.77-83,2008

[53] Y.Zhuang,W.Wang,R.Xing,"Target Tracking in Coloured Image Sequence Using Weighted Colour Histogram Based Particle Filter", Proceedings of the 2006 IEEE International Conference on Robotics and Biomimetics December 17 - 20, 2006, Kunming, China.

[54] J.Wang,Y.Yagi ,"Adaptive Mean-Shift Tracking With Auxiliary Particles",IEEE transactions on system ,man, and cybernetics—part B: cybernetics, pp1-12, 2009.

[55] J. Ning, L. Zhang, D. Zhang and C. Wu, "Robust Mean Shift Tracking with Corrected BackgroundWeighted Histogram", IET Computer Vision, 2010.

[56] R. T. Collins, "Mean-shift blob tracking through scale space", Proceedings of the IEEE Computer Society Conference on Computer Vision and Pattern Recognition, Vol. 2, pp. 234-240, 2003

[57] A. Yilmaz, "Object Tracking by Asymmetric Kernel Mean Shift with Automatic Scale and Orientation Selection", Proc. IEEE Conf. Computer Vision and Pattern Recognition, pp. 1-6, 2007.

[58] H. Qian, Y. Mao, J. Geng and Z. Wang, "Object tracking with self-updating tracking window", In PAISI, vol. 4430, pp. 82-93, 2007.

[59] Lankton S,Malcolm J,Nakhmani A, "Tracking through changes in scale", ICIP, pp.1-12, 2008.

[60] P.Pan,F.Porikli, "A new method for tracking performance evaluation based on a reflective model and perturbation analysis", ICASSP, 2009.

[61] J. Puzicha, Y. Rubner, C. Tomasi, and J. Buhmann, "Empirical evaluation of dissimilarity measures for colour and texture," in Proc. 7th Intl. Conf. on Computer Vision, Kerkyra, Greece, 1999, pp. 1165-1173.

[62] F.Porikli, "A Comprehensive Evaluation Framework and a Comparative Study for Human Detectors", IEEE transactions on intelligent transportation systems, vol.10, no.3, September 2009. 